\title{
Nellix Endovascular Aneurysm Sealing System (EVAS): A New Concept in Endovascular Repair - What the Radiologist Needs to Know
}

\section{Weller $A^{1 *}$, Shah $A^{2}{ }^{2}$, Seyed $A^{2}$, Touska $P^{2}$, Sayer $C^{2}$ and Vlahos $I^{2}$}

${ }^{1}$ The Institute of Cancer Research, Royal Marsden Hospital, Downs Road, Sutton, UK

${ }^{2} S t$ George's Hospital NHS Trust, London, UK

\begin{abstract}
The Nellix endovascular aneurysm sealing system (EVAS), licensed in Europe for treating infrarenal abdominal aortic aneurysms, has distinctive design comprising two balloon-expandable endoframes, each surrounded by a polymer-filled endobag.

This review describes the current role of CT in both Nellix follow-up and pre-procedure planning, with reference to the inclusion criteria for Nellix insertion and the deployment technique. Knowledge of the expected evolution of CT features following Nellix insertion is important for recognizing potential complications. Examples of normal post-operative CT appearance, as well as complications seen to date are discussed.
\end{abstract}

Keywords: Endovascular repair; Radiology; Abdominal aortic aneurysm; CT; Complications

\section{Introduction}

Clinical use of the Nellix endovascular aneurysm sealing system (EVAS) (Endologix, Santa Rosa, CA) for the treatment of infrarenal aortic aneurysms began in 2009 with promising early results. Until recently, all aortic endografts have had similar design morphology, typically of a bifurcated fabric-stent with proximal and distal attachments that fix and seal the device to the non-aneurysmal aorta and the iliac arteries. However, there are ongoing concerns regarding the long term outcomes of these devices, with migration, endo-leaks, aneurysm enlargement and rupture all potential risks [1-4]. Up to 2009, the infra-renal devices approved by the United States Food and Drug Administration (FDA) and European Medicines Agency (EMA) all achieved seal and longitudinal fixation through hooks, barbs, suprarenal stents, radial force and columnar rigidity [5-8].

In contrast, the sac anchoring EVAS comprises two balloon expandable stents that extend in parallel from the non-aneurysmal aorta proximally into the iliac arteries distally, each surrounded by a polymer filled endobag. The endobags obliterate the aneurysm lumen to achieve a seal and, in vitro and in theory, resist both lateral and longitudinal displacement forces. This device has FDA Investigational Device Exemption as well as European CE Mark approval and is currently undergoing clinical trial for efficacy [available at http://www. endologix.com/investigational_devices/nellix/clinical.php].

Although still undergoing evaluation for efficacy in infrarenal aneurysms with morphology suitable for conventional endovascular aneurysm repair (EVAR) devices, the Nellix device is potentially suitable for a wider range of aneurysmal anatomies than conventional EVAR systems. The aim of this pictorial review is to discuss the features of the Nellix EVAS, specifically with regard to the imaging findings post implantation that radiologists may encounter during follow-up imaging. Images of early complications after Nellix EVAS are also presented.

\section{Nellix Endografts Device Description and Insertion Procedure}

The Nellix EVAS comprises twin identical catheter based systems, one inserted on each side, and each with four components (Figure 1):

1. Two balloon expandable stainless steel endoframes maintain the aortic flow channel. The endoframes are placed in close opposition within the aneurysmal aorta, projecting into the neck proximally. Each extends into a common iliac artery distally.

2. A non-porous polyfluoroethylene (PTFE) - based endobag surrounds the endoframe and contains polymer. The endobags mould to the aneurysm sac lumen and provide a seal proximally and distally. Each fills via an injection system at the caudal end.

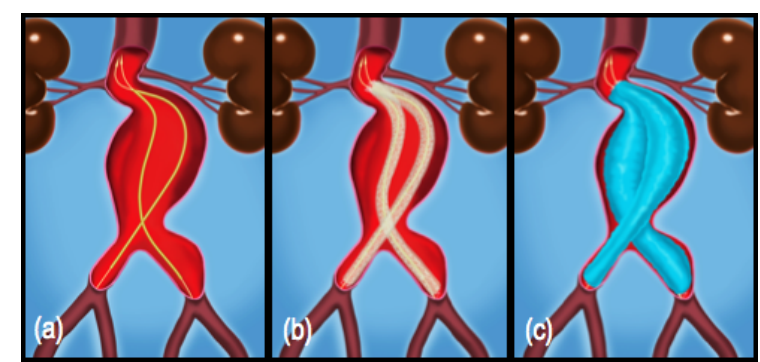

Figure 1: Nellix endovascualar sac anchoring system (EVAS) delivery, via two $17 \mathrm{Fr}$ tapered nose delivery catheters. Therefore catheters, containing endoframe and endobag, have ports for 0.035" guidewire insertion, angiography, balloon inflation and polymer injection. (a) 0.035 "guidewire insertion. (b) Catheters containing balloon expandable stainless stee endoframes, surrounded by a non-porous PTFE (polyfluoroethylene) endobag are deployed over the guidewire. The endoframe stents are balloon expanded. (c) The endobags are filled with biocompatible polyethyleneglycol (PEG) polymer, moulding to and filling the patent aneurysm sac lumen. The polymer injection system fills via tubing in the caudal end of the endobag, with monitors for fill volume and pressure.

*Corresponding author: Weller A, The Institute of Cancer Research, Roya Marsden Hospital, Downs Road, Sutton, UK, Tel: +44(0)7733115580; E-mail: alweller@gmail.com

Received February 23, 2016; Accepted March 04, 2016; Published March 11, 2016

Citation: Weller A, Shah AM, Seyed AR, Touska P, Sayer C, et al. (2016) Nellix Endovascular Aneurysm Sealing System (EVAS): A New Concept in Endovascular Repair - What the Radiologist Needs to Know. J Vasc Med Surg 4: 258. doi:10.4172/2329-6925.1000258

Copyright: ( 2016 Weller A, et al. This is an open-access article distributed unde the terms of the Creative Commons Attribution License, which permits unrestricted use, distribution, and reproduction in any medium, provided the original author and source are credited. 
Citation: Weller A, Shah AM, Seyed AR, Touska P, Sayer C, et al. (2016) Nellix Endovascular Aneurysm Sealing System (EVAS): A New Concept in Endovascular Repair - What the Radiologist Needs to Know. J Vasc Med Surg 4: 258. doi:10.4172/2329-6925.1000258

Page 2 of 6

3. Biocompatible polyethyleneglycol (PEG) based polymer fills the endobag and is mixed with iodinated contrast agent for visibility under fluoroscopy. The polymer, liquid when mixed, cures to a rubbery solid in $\leq 5 \mathrm{~min}$ at $37^{\circ} \mathrm{C}$. An injection system monitors fill volume and pressure.

4. $17 \mathrm{Fr}$ (external diameter) tapered nose delivery catheters, inserted via the femoral arteries, contain the endoframe, the surrounding endobag and ports for polymer injection, as well as ports for 0.035 " guidewire insertion, balloon inflation and angiography.

Device insertion is performed under fluoroscopic guidance (Figure 2) and once in position in the aorta, the two introduction catheters are simultaneously manipulated in the following steps [9]: (a) the endobag and fill-line integrity is confirmed by creating a vacuum through the system; (b) the stents are simultaneously balloon expanded; (c) mixed polymer solution is introduced to the endobags under volume and pressure monitoring; and (d) check

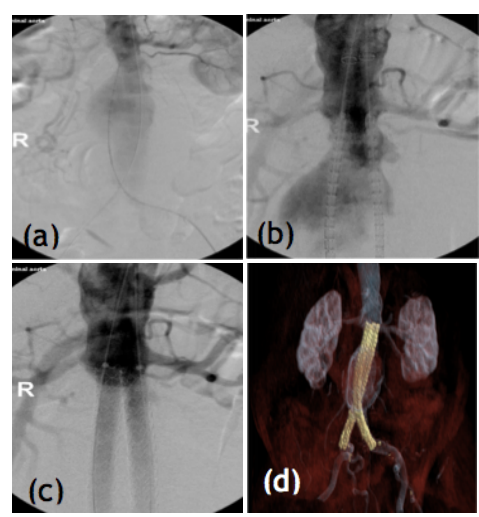

Figure 2: Nellix device insertion following introduction of the delivery catheters: ( $a$ and b) delivery catheters are introduced over 0.035 " guidewires (c) the endoframe stents are balloon expanded and polymer solution is introduced to the Endobags under pressure monitoring. (d) Post insertion 3-D volume rendered CT angiogram reconstruction. angiography performed to assess stent position and patency of renal vessels.

\section{Nellix Inclusion Criteria for Insertion and Anatomical Limitations}

The Nellix inclusion criteria covered by the manufacturer instructions for use (IFU) are as described in Table 1 and Figure 3 and include a greater range of infra-renal aortic aneurysm anatomies than other conventional EVAR devices are currently available [10]. However, unlike for conventional EVAR, juxta-and supra-renal aneurysms are not suitable for treatment with Nellix according to the current IFU and treatment of these aneurysms is not currently routine. The most commonly encountered anatomic limitation within the Nellix IFU for infra-renal aneurysms is a $60 \mathrm{~mm}$ maximum patent aneurysm lumen diameter, due to constraints in maximum endobag expansion. Despite this barrier, a recent retrospective survey of infra-renal EVAR showed Nellix to be suitable for up-to $70 \%$ of patients presenting for elective AAA repair, making Nellix applicable to a greater proportion of patients with infrarenal AAAs than other conventional stent grafts [9]. The main potential advantages of the Nellix device compared with conventional infrarenal devices are listed in Table 2.

\section{Pre-Procedure Planning and Follow up Imaging Protocol}

As for conventional EVAR, CT angiography is performed for preprocedure planning due to its exquisite anatomical detail. At this time, all patients eligible under the inclusion criteria for standard infrarenal EVAR may be considered for the Nellix device, although with minor differences in treatable infra-renal aortic aneurysm anatomies as described in Table 1 (different proximal neck diameter, length and angulation, common iliac artery diameter, length and tortuosity) With increasing experience of this device, these inclusion criteria may change, potentially enabling patients with more adverse neck and iliac artery anatomy to be treated with the Nellix EVAS. As stated previously, the flow lumen of the aneurysm should be evaluated and should be less than $60 \mathrm{~mm}$ diameter.

Rational imaging surveillance imaging post conventional EVAR is dictated by the need to detect endoleaks, device migration,

\begin{tabular}{|c|c|c|c|c|c|c|c|c|c|c|}
\hline Dims (mm) & $\begin{array}{c}\text { Gore } \\
\text { Excluder }\end{array}$ & $\begin{array}{l}\text { Cook } \\
\text { Zenith }\end{array}$ & $\begin{array}{c}\text { Gore } \\
\text { Excluder } \\
\text { Low Perm }\end{array}$ & $\begin{array}{l}\text { Endologix } \\
\text { Powerlink }\end{array}$ & $\begin{array}{l}\text { Cook Zenith } \\
\text { Enlarged Neck }\end{array}$ & $\begin{array}{l}\text { Medtronic } \\
\text { Talent }\end{array}$ & $\begin{array}{c}\text { Endologix } \\
\text { Enlarged Neck }\end{array}$ & $\begin{array}{l}\text { Gore Excluder } \\
\text { Enlarged Neck }\end{array}$ & $\begin{array}{l}\text { Summary of } \\
\text { EVARs }\end{array}$ & $\begin{array}{l}\text { Endologix } \\
\text { Nellix }\end{array}$ \\
\hline Neck Diam & $19-26$ & $18-28$ & $19-26$ & $18-26$ & $18-32$ & $18-32$ & $18-32$ & $19-29$ & $18-32$ & $16-32$ \\
\hline Neck Length & $\geq 15$ & $\geq 15$ & $\geq 15$ & $\geq 15$ & $\geq 15$ & $\geq 10$ & $\geq 15$ & $\geq 15$ & $\geq 10-15$ & $\geq 10$ \\
\hline Neck Angle & $\leq 60^{\circ}$ & $\leq 45^{\circ}$ & $\leq 60^{\circ}$ & $\leq 60^{\circ}$ & $\leq 60^{\circ}$ & $\leq 60^{\circ}$ & $\leq 60^{\circ}$ & $\leq 60^{\circ}$ & $\leq 45-60^{\circ}$ & $\leq 60^{\circ}$ \\
\hline $\begin{array}{l}\text { Iliac fixation } \\
\text { length }\end{array}$ & $\geq 10$ & $\geq 15$ & $\geq 10$ & $\geq 10$ & $\geq 15$ & $\geq 15$ & $\geq 15$ & $\geq 10$ & $\geq 10-15$ & $\mathrm{~N} / \mathrm{A}$ \\
\hline Iliac Diam & $10-18.5$ & $10-20$ & $10-18.5$ & $10-18.5$ & $8-18$ & $8-22$ & $10-23$ & $10-18.5$ & $8-23$ & $8-35$ \\
\hline
\end{tabular}

Table 1: Nellix instructions for use (IFU), compared with FDA and EMA approved conventional EVAR systems. Nellix manufacturer IFU include: short aneurysm neck beneath the renal arteries $(\geq 10 \mathrm{~mm})$; sharp angulation between aneurysm neck and lumen $\left(\leq 60^{\circ}\right)$; common iliac artery diameter 8-35 mm. The most common barrier to use is limited maximum aortic blood flow lumen diameter of $60 \mathrm{~mm}$.

\section{\begin{tabular}{l|l} 
Advantages & Disadvantages
\end{tabular}}

- Position at the top of the aneurysm sac rather than in the aneurysm neck for short - Anatomic limitation: Maximum $60 \mathrm{~mm}$ patent aortic aneurysm lumen diameter necked or sharply angulated necks (avoids neck angulation).

- Sac anchoring device, resisting lateral displacement and hence reduced migration risk in vitro. (constrained endobag expansion).

- The endobags seal side branch flow (lower risk of Type 2 endoleaks)

- Lack of data supporting use in juxta- and supra-renal aneurysms.

- Current uncertainty about the frequency and natural history of complications - long terms outcomes data is pending.

- Easier deployment with smaller diameter deployment catheters.

- Simplified treatment of common iliac artery aneurysms (obliterates aneurysm sac whilst preserving flow to EIA and IIA without need for IIA embolization).

Table 2: Nellix advantages and disadvantages over conventional EVAR. 
sac enlargement and possible rupture [11-14]. Experience with Nellix endografts is limited to relatively small reported case series, requiring further study before representative long term outcomes and complication rates are determined [15]. As a result, rational follow up imaging protocols are yet to be drafted. The surveillance imaging adopted by operators using the Nellix device has so far followed protocols used for conventional EVAR devices; computed tomography angiography (CTA) at 1, 6 and 12 months and annually thereafter.

\section{Initial Observations on Follow up Imaging}

On serial CT scans following uncomplicated Nellix EVAS, the most striking feature is a gradually reducing CT density of the endobag polymer over the first weeks to months post insertion (Figure 4). This happens at a variable rate. Immediately after the procedure, the Nellix PEG polymer is denser than sac thrombus, in keeping with a solid solution containing iodinated contrast medium introduced during deployment. Within 3-4 months, this overall increased density fades and a rim of high density emerges at the interface between the PEG polymer and hydrophobic surfaces (adjacent to the endobag gas locules and PTFE walls). In previous series, the reducing density was attributed to dissolution of iodinated contrast from the Nellix polymer $[16,17]$. However, in our experience, the concomitant developing high density rim surrounding the polymer suggests, rather than contrast dissolution; these changes represent iodinated contrast displacement from within the polymer to collect at the interface with PTFE or air.

Another striking feature following Nellix EVAS is of air locules that are frequently introduced to the endobag (and to a lesser extent into the aneurysm sac) during device deployment; these locules resorb within the first weeks, contemporaneous with the reducing CT density of the endobag polymer (Figure 4). On visual assessment of serial CT studies, the overlying endobag wall remains inflated in most cases and the resorbed air is replaced by material of density $20-30 \mathrm{HU}$, lower than the endobag polymer. However, in some cases, the endobag wall cannot be differentiated from underlying polymer and it is possible that the endobag collapses down into the defect previously occupied by air. It is at present unclear whether these latter features are associated with increased risk of type 1 or type 2 endoleaks.

A historic observation reported in 7 of the first 10 patients from the very first series of Nellix devices inserted, was of a non enhancing ring, or halo, located immediately between the endoframe and endobag on $\mathrm{CT}$ at 1 and 6 months. This finding was not associated with any change in aneurysm morphology or adverse clinical events. Following changes to the early endobag design, the halo was not seen in subsequent patients, suggesting that it reflected large endobag lumen diameter relative to the endoframe [17]. In our institution, using a more recent endobag design, we have not observed this feature.

\section{Initial Nellix Outcomes and Complications}

Initial clinical experience with Nellix, from 2009 to 2014, has yielded promising short-term results [17]. However, despite the potential suitability of a greater proportion of aneurysm anatomies for treatment within the Nellix IFU compared with conventional EVAR, current clinical experience and patient follow-up are limited. This requires larger case control studies for clinical and technical outcomes assessment before efficacy is confirmed and widespread adoption is justified.

Complications are most frequently diagnosed on follow-up imaging (with either Duplex ultrasound or CT) and their rates after conventional EVAR are known to be more common after treatment of adverse aneurysm anatomy, outside of the manufacturer IFU $[18,19]$. Early experience suggests that complications also occur using the Nellix device in patients where the proximal neck and distal iliac artery anatomy falls outside of the manufacturer's IFU [20]. Examples of complications after use of the Nellix device, more common in aneurysms that fall outside the IFU, are presented in Table 3.

Experience to date indicates reduced endoleaks occurrence, especially type 2, of Nellix compared with conventional EVAR. The Nellix endobag both anchors the device and obliterates the potential space for blood flow into the aneurysm sac. Only one type 2 endoleaks and two type 1 endoleaks have been identified in the literature to date. The type 2 endoleaks resolved spontaneously within 60 days of detection

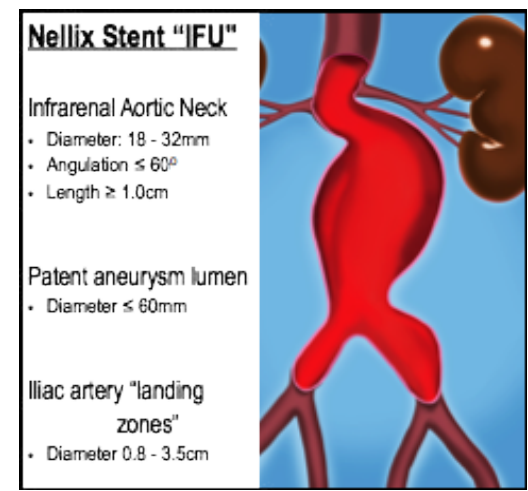

Figure 3: Parameters included in Nellix manufacturer instructions for use (IFU).
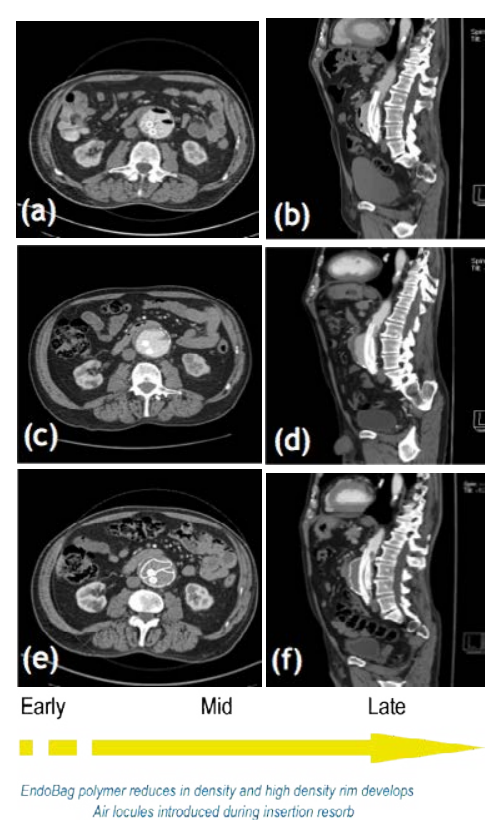

Figure 4: Normal CT appearances post Nellix stent insertion. (a and b) early appearance. Axial and sagittal images of CT aortogram performed 1 day postdeployment show homogenous high density and anti-dependent pockets of air within the endobag. (C-f) Mid-term and appearance. CT scans performed at 6 weeks (c and d) and 10 months (e and f) following stent insertion. These demonstrate gradual reduction in endobag PEG polymer density, development of high density rim surrounding the polymer and resorption of air locules introduced during insertion. In most cases, the endobag wall remains inflated and the resorbed air is replaced by material of density 20-30 HU. However, in some cases, it is possible that the endobag collapses down into the defect previously occupied by air. 
Citation: Weller A, Shah AM, Seyed AR, Touska P, Sayer C, et al. (2016) Nellix Endovascular Aneurysm Sealing System (EVAS): A New Concept in Endovascular Repair - What the Radiologist Needs to Know. J Vasc Med Surg 4: 258. doi:10.4172/2329-6925.1000258

[17]. Of the type 1 endoleaks, one proximal type la endoleaks resolved spontaneously between CT scans performed 1 and 2 months after insertion. The other, persistent distal type $1 \mathrm{~b}$ endoleaks (secondary to long aneurysm sac incompletely filled by the endobag), was successfully treated with an iliac extender and internal iliac artery embolization at 15 months $[15,20]$. Similarly, in our experience a simple covered iliac stent has been used to successfully treat a persistent iliac artery aneurysm following Nellix abdominal aortic aneurysm repair (Table 3 and Figure 5). The spontaneous resolution of some type 1 endoleaks following Nellix insertion, with no increase in sac size in the reported series to date, brings into question both the long term behavior of these endoleaks and hence their appropriate management (Figure 6).

Other potential complications include renal artery stenosis due to encroachment by the endobag (Figure 7), unilateral endografts luminal stenosis (Figure 8), and Nellix limb thrombosis (Figure 9) [18]. Currently, the frequency and long term behavior of endoleaks, limb kinking, partial renal artery occlusion and Nellix limb occlusion

\begin{tabular}{|c|c|c|}
\hline Complication observed & $\begin{array}{c}\text { Months } \\
\text { after device } \\
\text { insertion }\end{array}$ & Treatment \\
\hline Type 1a endoleak (Figure 6) & 3 & $\begin{array}{c}\text { Embolisation of endoleak } \\
\text { sac with Onyx }\end{array}$ \\
\hline $\begin{array}{c}\text { Renal artery stenosis following partial } \\
\text { occlusion by the endobag (Figure 7) }\end{array}$ & 2 & $\begin{array}{c}\text { Endovascular stenting of } \\
\text { the affected renal artery }\end{array}$ \\
\hline Nellix limb kinking distally (Figure 8) & Immediate & $\begin{array}{c}\text { Endovascular iliac limb } \\
\text { stent placement }\end{array}$ \\
\hline Nellix graft limb occlusion. (Figure 9) & 3.5 & None \\
\hline
\end{tabular}

Table 3: Examples of complications observed at our institution after Nellix EVAS.
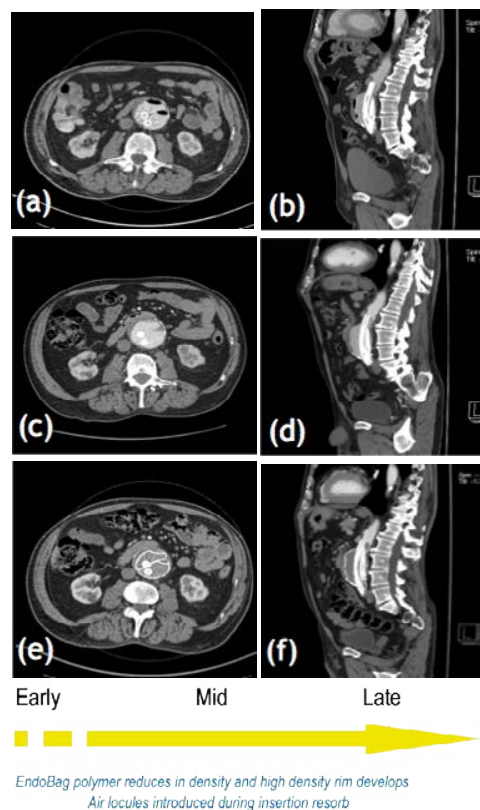

Figure 4: Normal CT appearances post Nellix stent insertion. (a and b) early appearance. Axial and sagittal images of CT aortogram performed 1 day postdeployment show homogenous high density and anti-dependent pockets of air within the endobag. (C-f) Mid-term and appearance. CT scans performed at 6 weeks (c and d) and 10 months (e and f) following stent insertion. These demonstrate gradual reduction in endobag PEG polymer density, development of high density rim surrounding the polymer and resorption of air locules introduced during insertion. In most cases, the endobag wall remains inflated and the resorbed air is replaced by material of density 20-30 HU. However, in some cases, it is possible that the endobag collapses down into the defect previously occupied by air.
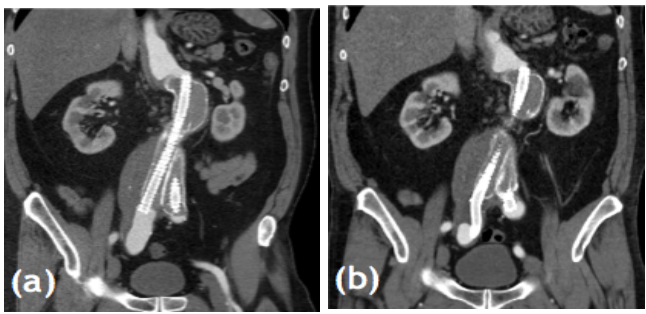

Figure 5: lliac artery extension of a right iliac artery aneurysm following Nellix treatment of an infrarenal abdominal aortic aneurysm. (a) Coronal CT aortogram demonstrates a successfully stented aortic aneurysm. (b) The known right common iliac aneurysm sac was subsequently covered with a right iliac endograft extension stent.
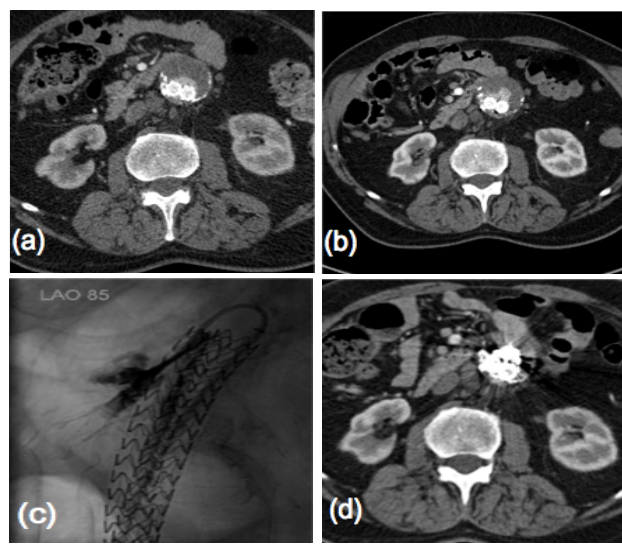

Figure 6: Type 1a endoleak. (a) Axial CT aortogram performed shortly after Nellix deployment. An area of high density anterior to the proximal graft may represent an endoleak or high density polymer within the Nellix endobag. (b) 3 months later, the polymer within the endobag has reduced in density and a small Type la endoleak is clearly evident. (c) Digital subtraction angiography image showing successful endovascular embolization of the endoleak with Onyx. (d) Subsequent axial CT aortogram demonstrates no residual filling of the endoleak, which is occluded with Onyx.

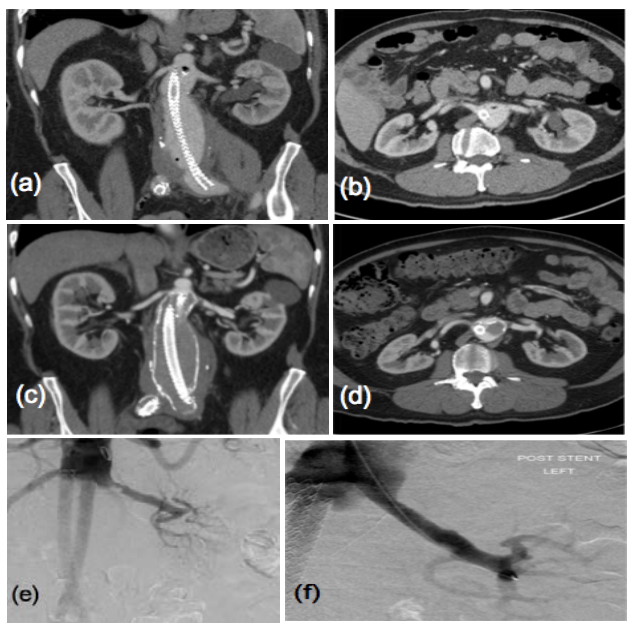

Figure 7: Renal artery stenosis following Nellix stent insertion. (aandb) Coronal and axial CT aortograms performed 2 days post-procedure demonstrate patent renal arteries on both sides. (candd) Corresponding CT images 2 months later show $>75 \%$ stenosis of the proximal left renal artery. (e) Conventional angiogram confirms stenosis of the proximal left renal artery, (f) successfully treated by endovascular stenting. 
Citation: Weller A, Shah AM, Seyed AR, Touska P, Sayer C, et al. (2016) Nellix Endovascular Aneurysm Sealing System (EVAS): A New Concept in Endovascular Repair - What the Radiologist Needs to Know. J Vasc Med Surg 4: 258. doi:10.4172/2329-6925.1000258

Page 5 of 6
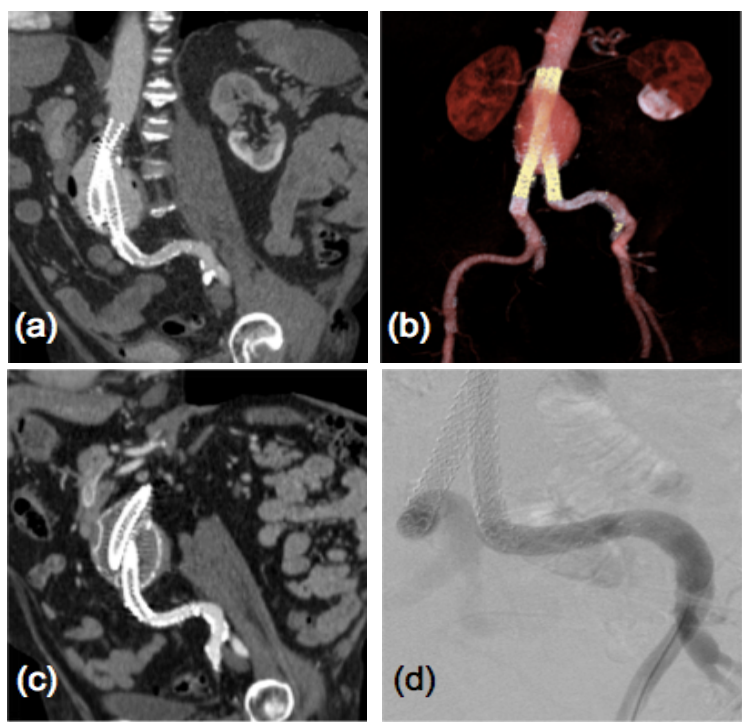

Figure 8: Nellix limb kinking distally. (a) Sharp angulation of the distal left Nellix is seen on this curved plane reconstruction and (b) confirmed on the 3-D reconstruction. Whether this kinked vessel would predispose to Nellix limb occlusion is unclear from the data available. (c and d) CT and digital subtraction angiography following endovascular stent insertion bridging the distal end of the Nellix (as a precautionary measure) confirm satisfactory opening of the kinked vessel lumen, with no pressure gradient measurable across the distal limb.
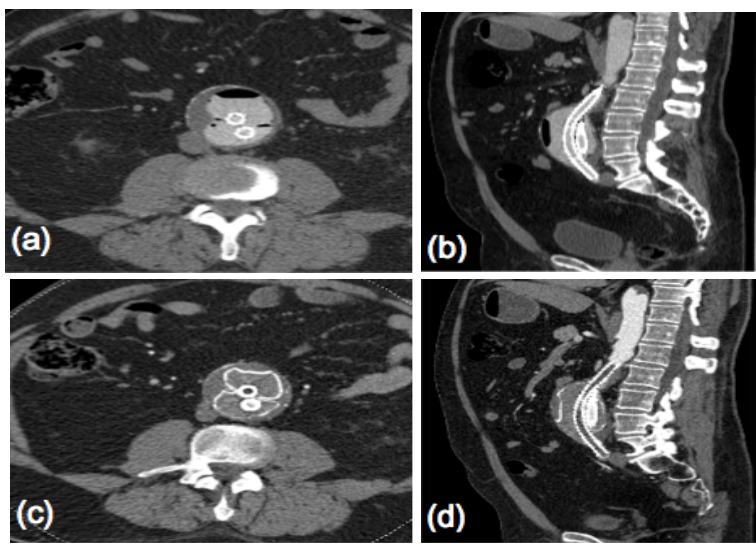

Figure 9: Nellix graft limb occlusion following insertion. ( $a$ and b) Axial and sagittal CT aortograms performed immediately post operatively show patent right and left endografts. Note high density polymer and anti-dependent air within the endobag, as expected. (c and d) Axial and sagittal CT images acquired 3.5 months later for routine follow-up show occluded left iliac endograft. The patient experienced increased claudicating on the left only. Note the previously discussed resorption of gas locules and reducing polymer density within the endobags with time.

following Nellix deployment compared with conventional EVAR is uncertain, due to lack of long-term outcome data and small number of patients in which complications have been seen.

\section{Conclusion}

The Nellix EVAS system is a relatively new EVAR device for patients with abdominal aortic aneurysms and has unique features compared with other conventional devices. Experience is ongoing and larger case control studies are required for outcomes assessment before efficacy is confirmed and widespread adoption justified. With increasing use of the Nellix EVAS, radiologists are likely to encounter patients undergoing follow-up CT scans after Nellix EVAR in their daily practice. In this pictorial review, we have presented the standard imaging features of this device and have presented illustrative examples of complications that can occur.

\section{References}

1. Laheij RJ, Buth J, Harris PL, Moll FL, Stelter WJ, et al. (2000) Need fo secondary interventions after endovascular repair of abdominal aortic aneurysms. Intermediate-term follow-up results of a European collaborative registry (EUROSTAR). Br J Surg 87:1666-1673.

2. Hobo R, Buth J, EUROSTAR collaborators (2006) Secondary interventions following endovascular abdominal aortic aneurysm repair using current endografts. A EUROSTAR report. J Vasc Surg 43: 896-902.

3. Kramer SC, Seifarth H, Pamler R, Fleiter T, Gorich J (2001) Geometric changes in aortic endografts over a 2-year observation period. J Endovasc Ther 8: 34-38.

4. Umscheid T, Stelter WJ (1999) Time-related alterations in shape, position, and structure of self-expanding, modular aortic stent-grafts: a 4-year single-center follow-up. J Endovasc Surg 6: 17-32.

5. Resch T, Malina M, Lindblad B, Malina J, Brunkwall J, et al. (2000) The impact of stent design on proximal stent-graft fixation in the abdominal aorta: an experimental study. Eur J Vasc Endovasc Surg 20: 190-195.

6. Heikkinen MA, Alsac JM, Arko FR, Metsänoja R, Zvaigzne A, et al. (2006) The importance of iliac fixation in prevention of stent graft migration. $J$ Vasc Surg 43: $1130-1137$

7. Arko FR, Heikkinen M, Lee ES, Bass A, Alsac JM, et al. (2005) Iliac fixation length and resistance to in-vivo stent-graft displacement. J Vasc Surg 41: 664-671.

8. Benharash P, Lee JT, Abilez OJ, Crabtree T, Bloch DA, et al. (2007) Iliac fixation inhibits migration of both suprarenal and infrarenal aortic endografts. J Vasc Surg 45: 250-257

9. Karthikesalingam A, Cobb RJ, Khoury A, Choke EC, Sayers RD, et al. (2013) The morphological applicability of a novel endovascular aneurysm sealing (EVAS) system (Nellix) in patients with abdominal aortic aneurysms. European Journal of Vascular and Endovascular Surgery 46: 440-445.

10. Karthikesalingam A, Holt PJ, Vidal-Diez A, Choke EC, Patterson BO, et al (2013) Predicting aortic complications after endovascular aneurysm repair. $\mathrm{Br}$ J Surg 100: 1302-1311

11. Ghatwary T, Karthikesalingam A, Patterson B, Hinchliffe R, Morgan R, et al (2012) St George's Vascular Institute Protocol: an accurate and reproducible methodology to enable comprehensive characterization of infrarenal abdominal aortic aneurysm morphology in clinical and research applications. J Endovasc Ther 19: 400-414.

12. De Bruin JL, Baas AF, Buth J, Prinssen M, Verhoeven EL, et al. (2010) Longterm outcome of open or endovascular repair of abdominal aortic aneurysm. N Engl J Med 362: 1881-1889.

13. United Kingdom EVAR Trial Investigators, Greenhalgh RM, Brown LC, Powell JT, Thompson SG, et al. (2010) Endovascular versus open repair of abdominal aortic aneurysm. N Engl J Med 362: 1863-1871.

14. Hobo R, Buth J (2006) Secondary interventions following endovascular abdominal aortic aneurysm repair using current endografts. A EUROSTAR report. J Vasc Surg 43: 896-902.

15. Kisis K, Krievins D, Naskovica K, Gedins M, Savlovskis J, et al. (2012) Quality of life after endovascular abdominal aortic aneurysm repair: Nellix sacanchoring endoprosthesis versus open surgery. Medicina (Kaunas, Lithuania) 48: $286-291$.

16. Donayre C, George E, Kopchok BS, Rodney A, White MD (2009) Fillable endovascular aneurysm repair. An early look at a next-generation EVAR technology that may address some current limitations and improve clinical outcomes.

17. Donayre CE, Zarins CK, Krievins DK, Holden A, Hill A, et al. (2011) Initia clinical experience with a sac-anchoring endoprosthesis for aortic aneurysm repair. J Vasc Surg 53: 574-582.

18. Karthikesalingam A, Nicoli TK, Holt PJ, Hinchliffe RJ, Pasha N, et al. (2011) The fate of patients referred to a specialist vascular unit with large infra-renal abdominal aortic aneurysms over a two-year period. Eur J Vasc Endovasc Surg 42: 295-301. 
Citation: Weller A, Shah AM, Seyed AR, Touska P, Sayer C, et al. (2016) Nellix Endovascular Aneurysm Sealing System (EVAS): A New Concept in Endovascular Repair - What the Radiologist Needs to Know. J Vasc Med Surg 4: 258. doi:10.4172/2329-6925.1000258

Page 6 of 6

19. Schanzer A, Greenberg RK, Hevelone N, Robinson WP, Eslami MH, et al. (2011) Predictors of abdominal aortic aneurysm sac enlargement after endovascular repair. Circulation 123: 2848-2855.
20. Krievins DK, Holden A, Savlovskis J, Calderas C, Donayre CE, et al. (2011) EVAR Using the Nellix Sac-anchoring Endoprosthesis?: Treatment of favourable and adverse anatomy. European Journal of Vascular and Endovascular Surgery 42: 38-46. 